\title{
THE ELECTROCARDIOGRAM OF POSTERIOR CARDIAC INFARCTION
}

\author{
BY
}

\author{
PETER MEYER
}

Received February 22, 1949

In suspected coronary artery disease, no clinical sign can as yet displace the electrocardiograph, which, by revealing past cardiac infarction, provides objective evidence for the diagnosis; much reliance must, therefore, be placed on the electrocardiographic signs of infarction. In lesions of the anterior wall, the signs are often characteristic, especially in chest leads. Posterior infarction is shown by deep and wide $Q$ deflections, by significant R-T deviation, and by $T$ inversion in lead II and III, and in the unipolar left leg lead. In the absence of coronary R-T changes, the signs of posterior infarction may be uncertain, since inversion of $T$ in lead III, and occasionally also in lead II, may occur without infarction, and Q in lead II and III and in the unipolar left leg lead may be equivocal. The practical experience of diagnostic difficulties has led to the present study which is to show the close similarity of certain types of cardiograms representing posterior infarction to tracings taken from patients without infarction, and to examine records of infarction for additional electrocardiographic signs that might be helpful in the interpretation of equivocal curves.

The object of the analysis was the form of the QRS complex, in which changes due to infarction could be expected to last longer, and to be more specifically characteristic of this lesion, than changes in the $\mathrm{T}$ wave. In the absence of necropsy control, care was taken to include cardiograms only if the clinical diagnosis was firmly established. Patients with cardiac infarction gave a typical history of pain, and showed the classical changes of posterior infarction in the standard leads. With one exception (Fig. 3F), cardiograms were rejected if the signs of infarction were confined to the R-T segment and the $T$ wave and did not include a small $Q$ in lead III; if the cardiogram was equivocal, the diagnosis was accepted only if it was confirmed by serial tracings. Infarction was held to be absent if there was no history of pain and if the age and clinical condition provided no suspicion of coronary disease; in a number of cases, the clinical diagnosis was further supported by the presence of changes in serial cardiograms. Most of the tracings reviewed were taken in private practice with a Siemens electrocardiograph which registers time in 0.05 second; some of the patients were seen at the National Heart Hospital, under the care of Dr. William Evans. The records of 40 patients with posterior infarction were analysed.

\section{Electrocardiograms with Equivocal Changes OF THE QRS COMPLEX IN THE STANDARD LEADS}

Of 40 patients with posterior infarction, 7 had standard limb leads with $Q$ deflections in lead III, or II and III, which did not differ in size or depth or both from similar $\mathbf{Q}$ waves seen in patients without infarction. The cardiographic signs that caused uncertainty in the interpretation of the curves varied with the position of the long axis of the heart. In concordant cardiograms, showing mainly upright QRS complexes in the standard leads as seen when the heart was in the "vertical" position according to Wilson's terminology, Q II and Q III were of uncertain significance if they were less than 0.04 second in width and if the amplitude of Q III was less than 25 per cent of the tallest $R$ in any limb lead. Cardiograms with a similar QRS pattern were seen in patients without infarction. In the tracings illustrated in Fig. 1 the infarct could not be recognized by the appearance of the $Q$ waves or by any other change in the QRS complex; the presence or absence of $S$ waves in lead I had no relation to the diagnosis, but Q I was more likely to be present when there was no infarction.

In discordant cardiograms, with QRS mainly upright in lead I and downward in lead III, Q was equivocal if it was deep, or deep and wide, in lead III, but small or absent in lead II. Care had to be taken to exclude a false Q III: when each successive beat in lead III was examined, a small $R$ was often 


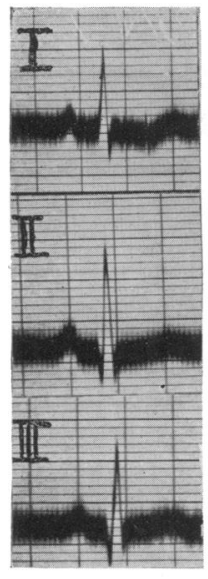

A

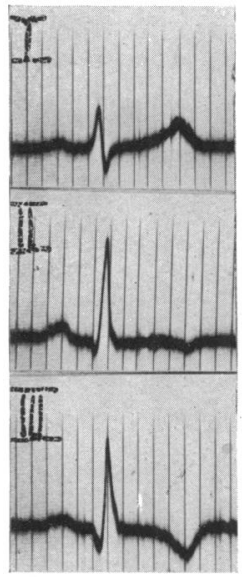

E

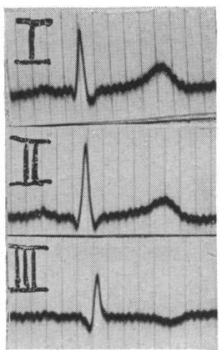

B

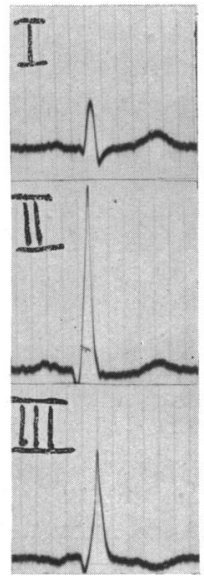

F

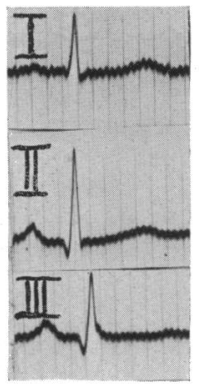

C

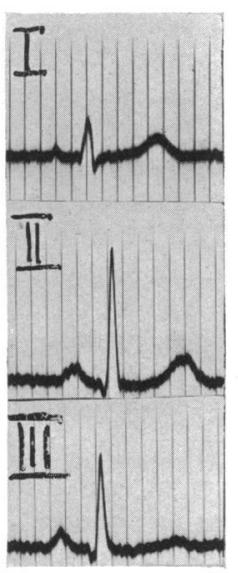

G

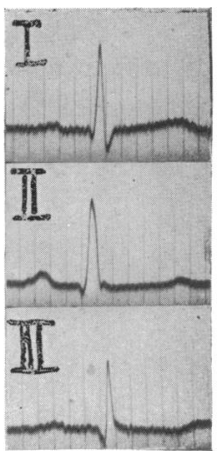

D

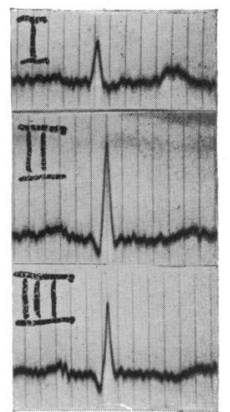

H

FIG. 1.-Electrocardiograms of the concordant type with equivocal $\mathrm{Q}$ deflections.

(A) Case 1: Man aged 45: posterior infarction (see also Fig. 4C).

(B) Case 2: Man aged 36: palpitation.

(C) Case 3: Man aged 77: broncho-pneumonia.

(D) Case 4: Man aged 47: palpitation.

found to precede the downward deflection which, in many tracings, was then revealed as an $\mathbf{S}$ wave. The absolute size of Q III and its size relative to the $\mathbf{R}$ waves in other leads, was the same in patients with and without infarction; a wide Q III was more frequent in infarction (Fig. 2A and E) but also occurred in its absence (Fig. 6B); a Q I was again recorded more often when there was no infarction.

Deep inspiration decreased the size of Q III in most patients with infarction and in normal controls
(E) Case 5: Man aged 47: posterior infarction.

(F) Case 6: Man aged 31: left mammary pain.

(G) Case 7: Woman aged 21: extrasystoles.

(H) Case 8: Woman aged 57: thyroid toxæmia.

(Fig. 5 and 6). Infrequently, Q III was abolished by deep breathing in subjects without infarction (Fig. 5C and 6B). In infarction Q I was usually absent or small. If in individual patients $Q \mathbf{I}$ was shown before infarction, it was reduced in amplitude or abolished after the attack (Fig. 3); but when the cardiograms of all patients with posterior infarction were examined, Q I was found in 14 out of 40 records. The presence or absence of $Q I$ was, therefore, of no diagnostic significance. 


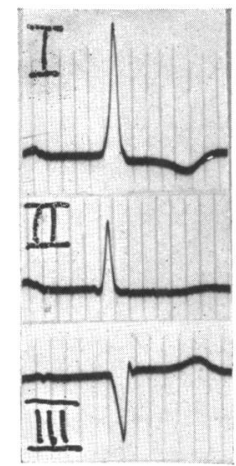

A

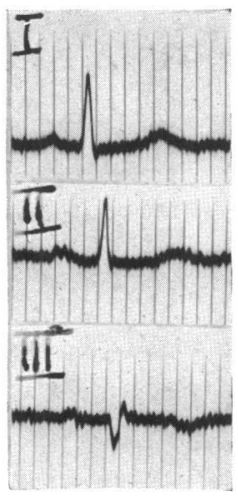

B

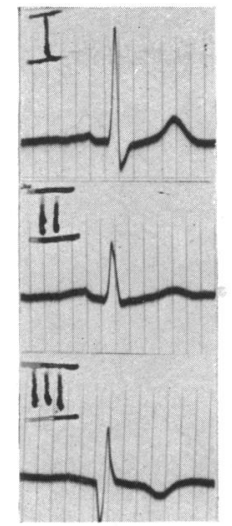

$\mathrm{C}$

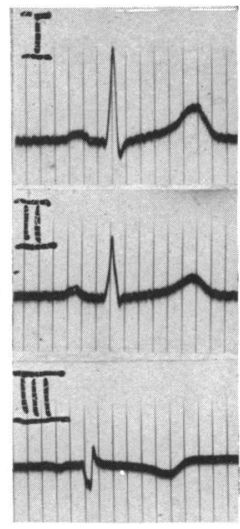

D
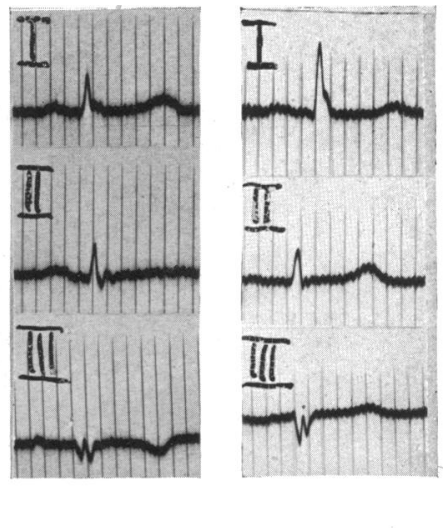

$\mathrm{E}$
(A) Case 9: Man aged 67: hypertension and posterior infarction (ten years after the tracing shown in Fig. 3A).

(B) Case 10: Man aged 50: left mammary pain.

(C) Case 11: Woman aged 65: moderate hypertension.

\section{UNipolar Limb Leads IN Posterior CARDIAC INFARCTION}

Unipolar limb leads were analysed in 18 cases of posterior infarction. The leads were taken with the Wilson technique and then designated VR, VL, VF. In other cases, the Goldberger method was adapted to the central terminal of Wilson by detaching the indifferent limb electrode from the limb under exploration; the leads were then called aVR, aVL, and aVF. The left leg lead showed a deep and wide $Q$ (with an amplitude of 30 per cent or more of the amplitude of $R$ in this lead and with a width of 0.04 second or more) when Q II and Q III were also characteristic (Fig. 4). In cardiograms with discordant standard leads and equivocal $Q$ waves, $Q$ in the left leg lead was wide (Fig. 6A), but not always deep (Fig. 6D); in records of the concordant type with small Q waves in lead II and III, $\mathrm{Q}$ in the left leg lead was also small in width and size (Fig. 5A and D). In all cases of infarction, the Q wave, whatever its size, was deeper in lead III than in the left leg when allowance was made for augmentation in Goldberger leads. The voltage of $Q$ in the left leg lead was reduced by deep inspiration.

All cases of posterior infarction showed an initial upright deflection in the left arm lead; it was more than $1 \mathrm{~mm}$. in amplitude and often broad or slurred in the ascending limb. A monophasic upright deflection was recorded in some cases; others showed an $\mathbf{S}$ wave, but a small $\mathbf{R}$ followed by a deep $\mathbf{S}$ was not
(D) Case 12: Woman aged 37: extrasystoles.

(E) Case 13: Man aged 66: posterior infarction (one year after the tracing shown in Fig. 3H).

(F) Case 14: Woman aged 44: left mammary pain.

seen. In the right arm lead, the initial deflection was upright in 15 out of 18 cases and varied in amplitude from a small spike to a size of $4 \mathrm{~mm}$.; an $R$ wave in the right arm would be expected to show as a $Q$ wave in lead $I$, but in all 15 cases $Q I$ was either absent or smaller than $R$ in the right arm lead after allowance was made for augmentation. When right bundle branch block was added to posterior infarction, the unipolar limb leads conformed to the infarction pattern (Fig. 4D).

\section{Unipolar Limb Leads in Patients without INFARCTION SHOWING CARDIOGRAMS WITH InCoNClusive $Q$ Waves IN THE Standard LEADS}

Equivocal $\mathbf{Q}$ waves in the standard leads did not signify infarction if the initial deflection in the left leg lead was upright (Fig. 5C and 6B); in patients of this type, Q III was abolished by deep inspiration. In concordant cardiograms, a small $Q$ in the left leg lead was as inconclusive as a small Q in lead II and III; it was not abolished by deep inspiration. Unlike the $Q$ of infarction, Q VF was often of the same size as $Q$ III (Fig. 5E and F). In these cases, the left arm lead also differed from the infarction pattern and showed an $R$ wave of small voltage. If $Q$ II and Q III were equivocal and the cardiogram conformed to the infarction pattern in lead VF and VL, a significant deviation might yet be shown in the relation of $Q I$ to the right arm lead: in this case, $Q$ I was of the same size as $R$ in VR (Fig. 5B). In 


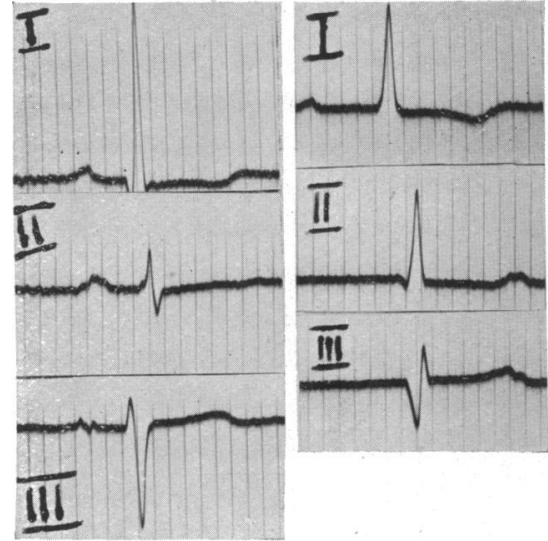

A
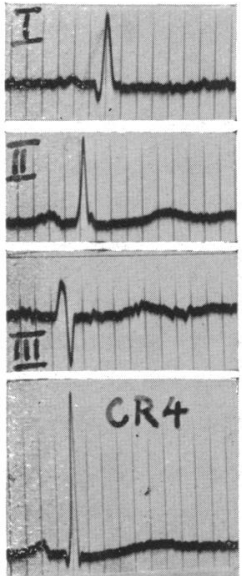

E

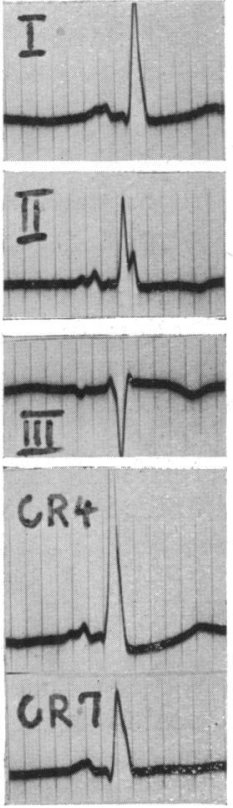

$\mathrm{F}$

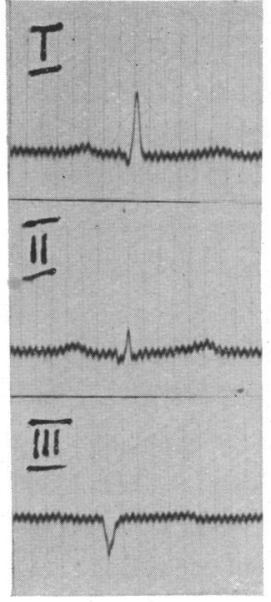

C
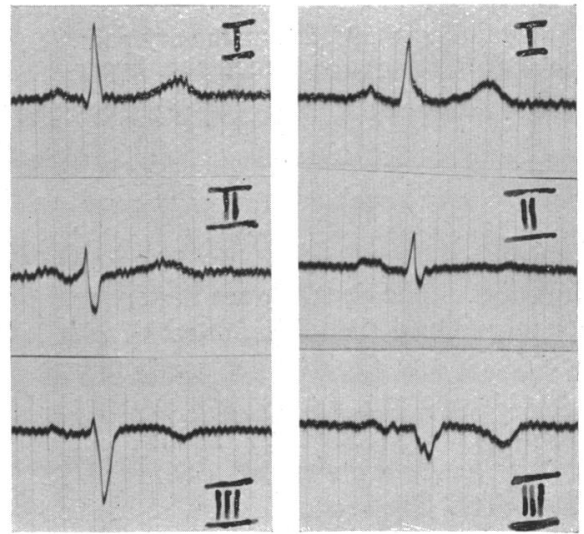

Fig. 3.-Electrocardiograms taken before and after posterior infarction.

(A) Case 9: Man aged 57: hypertension.

(B) The same: two years after infarction.

(C) Case 15: Man aged 58: angina of effort.

(D) The same: three weeks after infarction.

discordant cardiograms, $Q$ in the left leg lead was less wide than the $Q$ of infarction; it differed from the $Q$ VF seen in the concordant type of tracing in that it was deep, yet smaller than Q III, even in the absence of infarction. An initial downward
(E) Case 16: Woman aged 67: hypertension.

(F) The same: on day of infarction.

(G) Case 13: Man aged 65: angina of effort.

(H) The same: two months after infarction.

deflection in the left arm lead occurred only in patients without an infarct (Fig. 6E).

It was concluded that equivocal $Q$ waves in the standard leads did not indicate posterior infarction if in unipolar limb leads the initial deflection was 


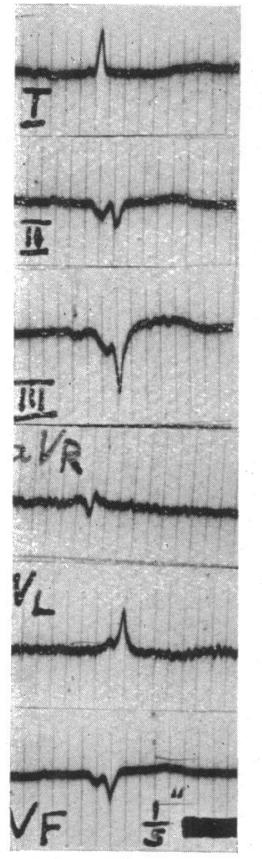

A

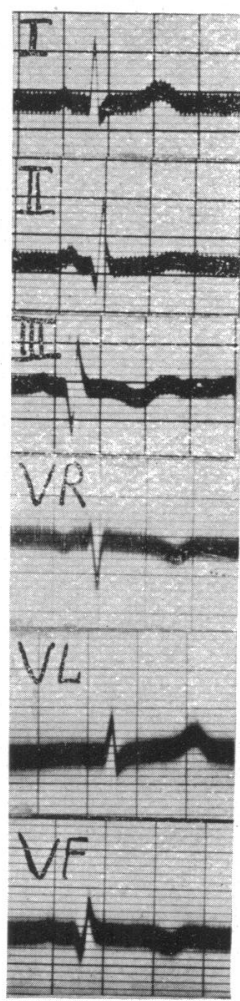

B

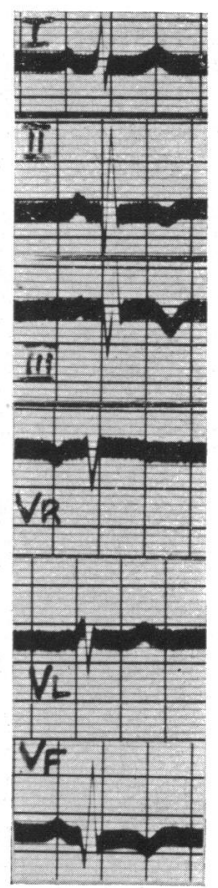

C

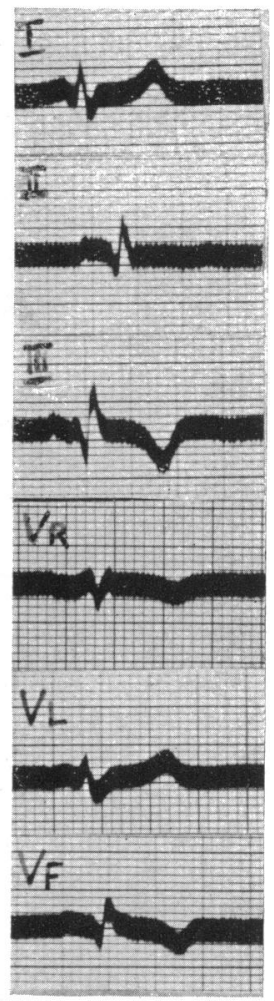

D

Fig. 4.-Unipolar limb leads in cardiograms with characteristic changes in the standard leads.

(A) Case 17: Man aged 61: infarction three years ago.

(B) Case 18: Woman aged 39: infarction two years ago.

upright in the left leg, or downward in the left arm, or if an initial $\mathrm{R}$ shown in the right arm was also recorded in lead $I$ as a $\mathbf{Q}$ of similar size; $a \mathbf{Q}$ in the left leg did not represent infarction if it was of the same amplitude as Q III. When the standard leads were concordant, equivocal $Q$ waves in lead II and III were suggestive of infarction if $Q$ in the left leg lead was smaller than Q III and the right and left arm leads conformed to the infarction pattern. In discordant tracings, care had to be taken to ascertain that the downward deflection in lead III was a $\mathbf{Q}$ and not an S wave. A true Q III stood for infarction if $Q$ in the left leg lead was 0.04 second or more in width; a deep Q, even when smaller than Q III, did not indicate an infarct in this type of tracing. If Q III was abolished by deep inspiration, absence of $Q$ in lead VF could be inferred and infarction was unlikely; the effect of deep inspiration was inconclusive if it left Q III unchanged or reduced in size.
(C) Case 1: Man aged 45: infarction three weeks ago (10 days after the tracing shown in Fig. 1A).

(D) Case 19: Man aged 54: infarction nine weeks ago.

\section{Discussion}

The preceding observations have shown the unipolar limb leads to be of value in the cardiographic diagnosis of posterior infarction. In the past, the unipolar left leg lead was singled out for examination in cases of posterior infarction, and the diagnostic significance of deep and wide $Q$ deflections in this lead was stressed (Myers and Oren, 1945; Goldberger, 1947). Although such Q deflections were found in the present series of cases, difficulties arose because there were patients with infarction who exhibited a small and short $Q$, and subjects without infarction with deep $Q$ waves in lead VF. When such equivocal cardiograms were studied, examination of the other unipolar limb leads was valuable.

On theoretical grounds, changes in the curves of the left and right arm must be expected with the development of $\mathrm{a} Q$ in the left leg lead due to 

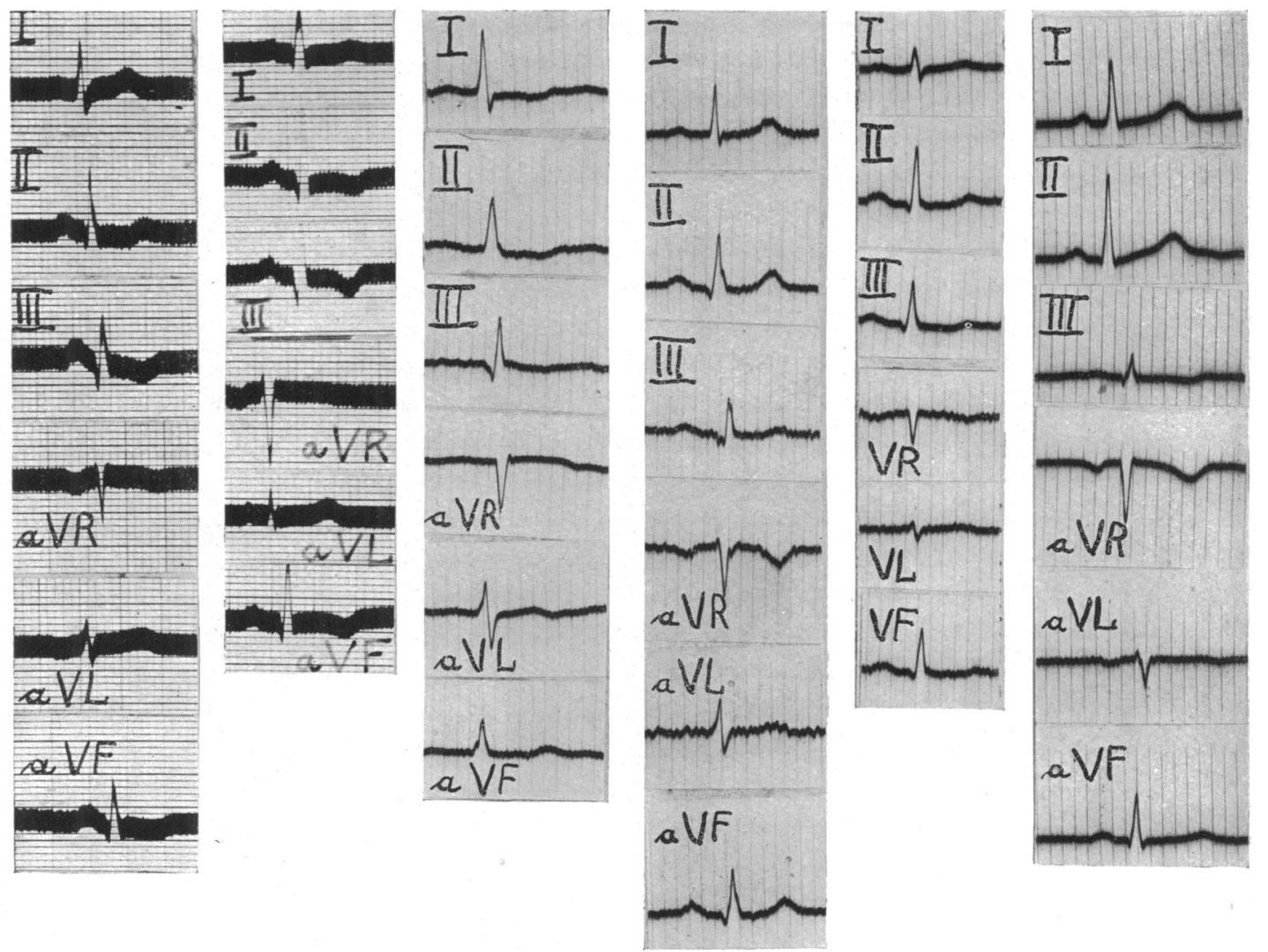

II
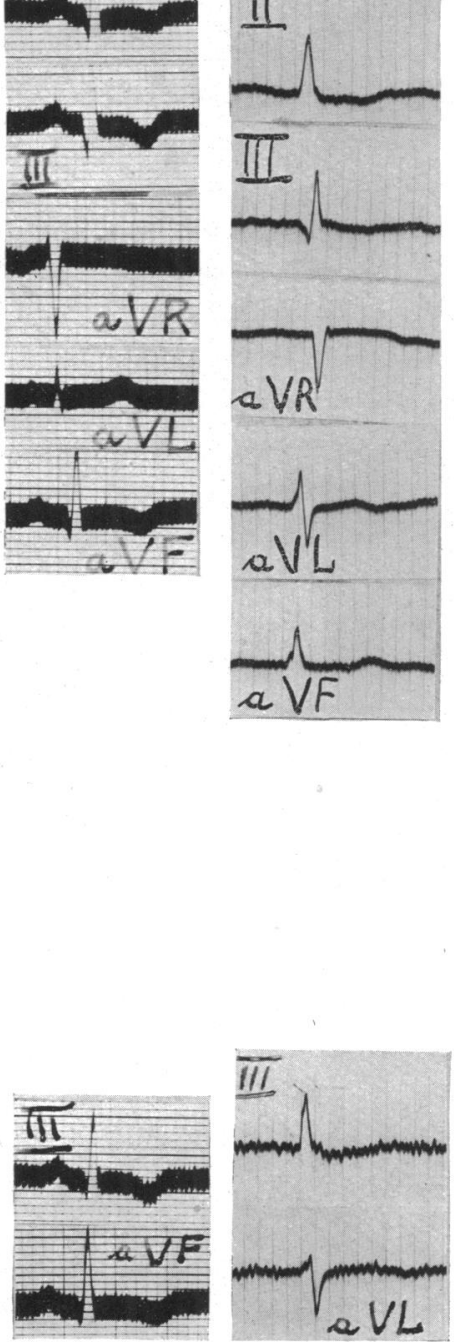

A

C

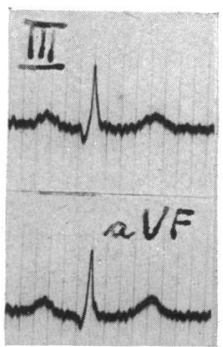

D

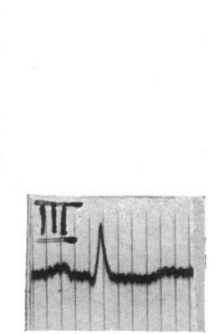

$\mathrm{E}$

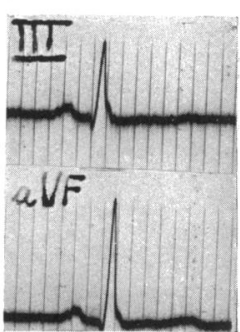

F

Fig. 5.-Unipolar leads in cardiograms with equivocal $Q$ deflections in the standard leads (concordant type). Lower row: Leads taken on deep inspiration.

(A) Case 20: Man aged 49: infarction nine months ago.

(B) Case 21: Man aged 38: repetitive paroxysmal tachycardia.

(C) Case 22: Woman aged 36: mitral stenosis, aortic incompetence, auricular fibrillation.
(D) Case 23: Man aged 53: infarction one year ago.

(E) Case 24: Man aged 64: emphysema and bronchitis.

(F) Case 25: Woman aged 47: hypertension 

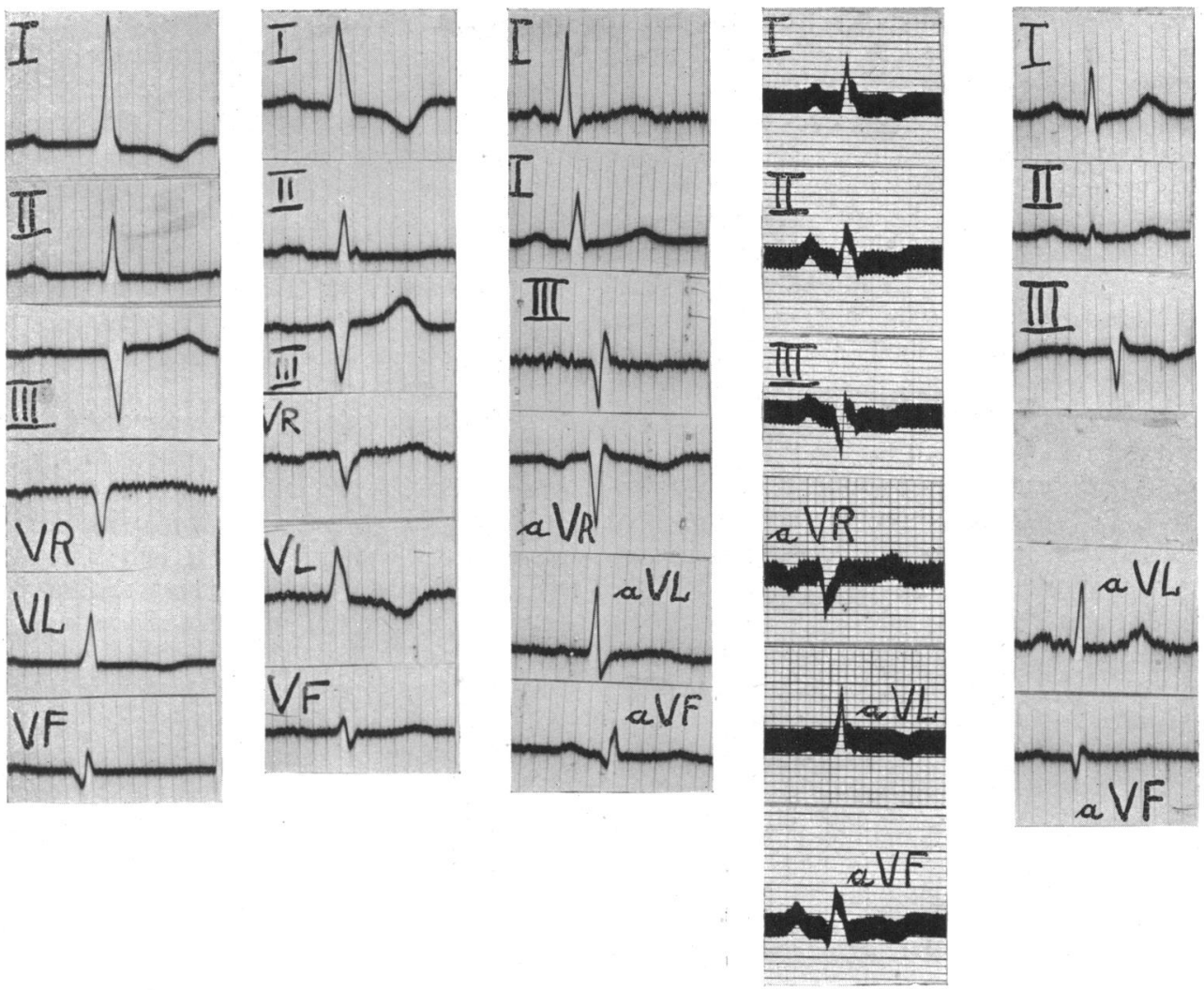

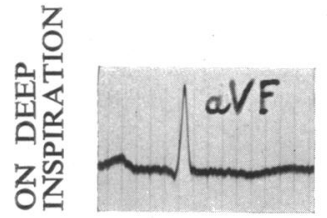

A

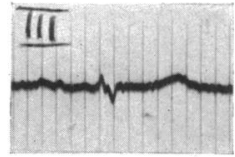

B

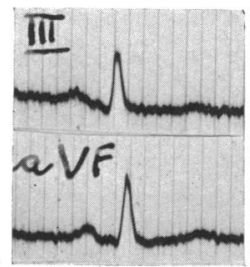

$\mathrm{C}$

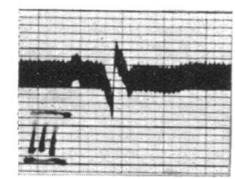

$\mathrm{D}$

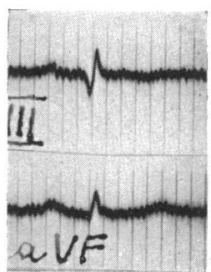

E

FIG. 6.-Unipolar leads in cardiograms with equivocal Q deflections in the standard leads (discordant type).

Lower row: Leads taken on deep inspiration.

(A) Case 9: Man aged 57: hypertension and posterior infarction (see also Fig. 2A, 3A and $B)$.

(B) Case 26: Man aged 63: hypertension.

(C) Case 27: Man aged 57: hypertension.

infarction as the sum of the potentials in the three limbs leads must remain zero at any given time. Examination of the standard leads had shown that a Q I present before infarction is reduced in size or abolished by the infarct; since such $Q$ waves usually derive from an initial negative deflection in the left arm lead, it was expected that the development of
(D) Case 28: Man aged 49: infarction four weeks ago.

(E) Case 29: Woman aged 69: moderate hypertension.

early negativity in the left leg lead from infarction would cause early positivity in the left arm lead, and this has been confirmed by observation. As the upward deflection in the left arm lead is a counterpart of $Q$ in the left leg lead, it follows that it must occur early in ventricular excitation and be synchronous with $Q$ in the left leg lead. Lead I 
measures the potential difference from the left to the right arm; a $Q$ in the left arm or an $R$ in the right arm will be recorded as a $Q$ in lead $I$. Since early negativity in the left arm does not occur in these cases of posterior infarction, a $Q$ in lead I can only be due to early positivity in the right arm; but the positive deflection in the left arm must partly or wholly counteract the formation of a $\mathrm{Q}$ in lead $\mathrm{I}$ from the positive deflection in the right arm; therefore, $\mathbf{Q} I$ is smaller than $R$ in $V R$, or it is absent. Observation has confirmed this argument. In the absence of infarction, a $Q$ in the left leg lead is not necessarily combined with a prominent $R$ in the left arm lead: the initial deflection may be of low voltage (Fig. 5E and F) or may be directed downward (Fig. 6E). There is also the possibility that $\mathbf{R}$ in the left arm lead may not occur early in ventricular excitation: this can be recognized if the $\mathbf{R}$ wave does not interfere with the formation of a $\mathbf{Q} I$ from $\mathbf{R}$ in the right arm lead. Such a combination of events has been seen in one record (Fig. 5B).

When the standard leads are concordant, infarction is likely if Q III is larger than Q in the left leg lead. This is an indirect method of observing the early upright deflection in the left arm which reinforces the downward direction of Q III initiated by $Q$ in the left leg; in the absence of infarction, the small amplitude of $R$ in the left arm prevents this reinforcement of $Q$ III. Discordant cardiograms usually show a prominent $R$ in the left arm lead; hence Q III is larger than Q in VF also in the absence of infarction. Deep inspiration, by causing a shift of the long axis of the heart away from the left arm, reduces the amplitude of $R$ in VL (Fig. 5C); it therefore abolishes the $Q$ waves which are not due to $\mathbf{Q}$ in the left leg lead.

In the left leg lead, a $\mathbf{Q}$ of infarction may be small in size and duration in concordant tracings. It is wide though not necessarily deep in cardiograms of the discordant type, when a wide and deep Q III is also shown; but a wide and deep $Q$ in lead III alone does not stand for infarction because it also occurs from left ventricular hypertrophy (Myers and Oren (1945); also Fig. 6B).

\section{SUMmary AND CONClusion}

The electrocardiograms of 40 patients with posterior infarction were examined for modifications of the QRS complex indicating infarction; there were 7 cases with inconclusive Q waves in lead III, or II and III, and they closely resembled cardiograms from subjects without infarction. In concordant tracings, uncertainty was caused by small $Q$ waves in leads II and III; in the discordant type, difficulties arose when $Q$ was deep, or wide and deep, in lead III, and small or absent in lead II; care had to be taken to examine each beat in lead III to ascertain that the downward deflection in this lead was not in fact an $\mathbf{S}$ wave. A $\mathbf{Q}$ wave in lead I was more often seen in normal subjects than after infarction, but this was of no diagnostic significance; in the individual patient, such a $Q$ wave shown before infarction was reduced in amplitude or abolished by the infarct.

Unipolar limb leads were examined in 18 cases of posterior infarction with $Q$ waves in the standard leads. The left leg lead showed a $Q$ deflection in all cardiograms; it was deep and wide if typical $Q$ waves were seen in leads II and III. When the standard leads were discordant with a wide and deep Q III, Q in the left leg lead was wide, but not always deep. When the standard leads were concordant with inconclusive $\mathbf{Q}$ waves in leads II and III, $Q$ was also small in the left leg. In all records of plain posterior infarction, Q III was larger than $Q$ in the left leg; the left arm lead showed an initial upright deflection of more than $1 \mathrm{~mm}$.; in most, but not all records, an initial upright deflection was also shown in the right arm, and this did not appear as a $Q$ wave of the same size in lead $I$.

From an examination of unipolar limb leads in subjects without infarction it was concluded that equivocal $Q$ waves in the standard leads did not indicate plain posterior infarction if the initial deflection was upright in the left leg, or downward in the left arm, or if an initial $R$ shown in the right arm was also recorded in lead $\mathrm{I}$ as a $\mathrm{Q}$ of similar size. A $\mathbf{Q}$ wave in the left leg lead did not represent infarction if it was of the same amplitude as Q III. When the standard leads were concordant, equivocal $Q$ waves in leads II and III were suggestive of infarction if $Q$ in the left leg lead was smaller than Q III and if right and left arm leads conformed to the infarction pattern. In discordant tracings, the width of the $Q$ wave in the left leg lead was significant; a deep Q wave, even when smaller than $\mathbf{Q}$ III, did not indicate an infarct. The effect of deep inspiration on the $Q$ wave in lead III and lead VF was also examined; if Q III was abolished by deep inspiration, absence of $Q$ in lead VF could be inferred.

In the discussion, the electrical events leading to the observed cardiographic patterns were analysed.

I wish to record my gratitude to Dr. William Evans for much advice and encouragement received from him.

\section{REFERENCES}

Goldberger, E. (1947). Unipolar Lead Electrocardiography. London, Henry Kimpton.
Myers, G. B., and Oren, B. G. (1945). Amer. Heart J., 29, 708 . 